\title{
Correlates and clinical implications of tic suppressibility
}

Keisuke Ueda, $\mathrm{MD}^{1}$, Soyoung Kim, $\mathrm{PhD}^{2}$, Deanna J. Greene, $\mathrm{PhD}^{3}$, Kevin J. Black, $\mathrm{MD}^{1,2,4,5}$

1. Department of Neurology, Washington University School of Medicine, St. Louis, MO, USA

2. Department of Psychiatry, Washington University School of Medicine, St Louis, MO, USA

3. Department of Cognitive Science, University of California San Diego, La Jolla, CA, USA

4. Department of Radiology, Washington University School of Medicine, St Louis, MO, USA

5. Department of Neuroscience, Washington University School of Medicine, St Louis, MO, USA

Corresponding authors:

Keisuke Ueda, M.D.

660 S Euclid Avenue

Campus Box 8111

St. Louis, MO 63110-1093

Phone: 314-454-6120, Fax: 314-454-2523

Email:k.ueda@wustl.edu

ORCID: https://orcid.org/0000-0003-4087-6018

Number of words: about 3468 words (2000-4000)

Number of references: 95

Number of figures: 0

Number of tables: 0

Key words (up to 6 words): Tourette syndrome [MeSH]; provisional tic disorder; suppression; Inhibition, psychological [MeSH]; tic disorders [MeSH]

Human/Animal rights or Informed Consent statement: N/A 
Abstract: (131/150)

Purpose of review: Tic disorders are common in the pediatric population and are differentiated from other movement disorders by tic suppressibility. Understanding the mechanism of tic suppression may provide new insights to the pathophysiology of tic disorders. This article highlights clinical phenomenology and neuronal correlates of tic suppressibility.

Recent findings: Recent studies suggest that tic suppressibility exists in children shortly after onset of their tics. Moreover, those who are better able to suppress their tics have better tic outcomes. Interoceptive awareness and automatic action inhibition may be involved in tic suppression.

Summary: We illustrate a possible underlying mechanism of tic suppressibility and its clinical correlations and implications. New concepts such as interoceptive awareness and action inhibition may help explain tic disorders. Further study will be useful to fill remaining knowledge gaps. 


\section{Introduction}

Tics, defined as "sudden, rapid, recurrent, nonrhythmic motor movement or vocalization" [1], are one of the symptoms of hyperkinetic movement disorders. Tics are prevalent in the pediatric population with at least $20-25 \%$ of elementary school children exhibiting motor tics [2-4]. The onset of tic disorders must occur before the age of 18 years to be considered primary (i.e., not explained by other disorders like Huntington's disease). Provisional Tic Disorder is diagnosed when the duration of tics is less than one year. After one year, Tourette's Disorder or Persistent Tic Disorder (collectively hereafter, Tourette syndrome: TS) can be diagnosed. Tics usually begin between 3 and 8 years of age and show a waxing and waning course with peak tic severity occurring around 8 to 12 years of age. Many patients experience improvement of their tics by early adulthood [5].

Hyperkinetic movement disorders (e.g., chorea, dystonia, and tic disorders) are conceptualized as disorders of the basal ganglia with abnormal inhibitory networks leading to a failure to inhibit unwanted movements [6]. Their various motor symptoms depend on the dysfunction of inhibitory networks. Unlike other hyperkinetic movement disorders such as dystonia or myoclonus, patients with tics frequently experience premonitory urges (PU), defined as an unpleasant sensation or perceived need to move that precedes tics and is temporarily relieved by the execution of tics [7-9]. Patients with tics can suppress their tics for variable periods of time, which has contributed to their being described by some as "un- 
voluntary" $[10,11]$. This suppressibility can differentiate tics from other movement disorders and has therapeutic implications through behavioral therapies. A questionnaire survey regarding daily experience with tic suppression revealed that $58 \%$ of TS children and adults attempted to suppress their tics in daily life, but were not always successful [12]. Sometimes they felt uncomfortable or incomplete when they suppressed tics and found tic suppression difficult (i.e. requiring concentration or extra effort). Thus, effective tic suppression can be closely tied to quality of life, and unsuccessful tic suppression may lead to difficulty in schoolwork, negative responses from peers or teachers, and decline in selfesteem [13]. Understanding the pathophysiology of tic suppressibility is clinically beneficial and may inform tic pathology and the natural course of tic disorders.

In this paper, we review the existing literature to describe clinical characteristics and brain correlates of tic suppressibility. In particular, we highlight recently published studies, aiming to create an up-to-date overview of tic suppressibility.

\section{Clinical phenomenology of tic suppressibility}

Generally, both motor and vocal tics are suppressible to a degree [14]. In clinical observation studies, children with tics were able to suppress their tics by nearly $70 \%$ in frequency for as long as 40 minutes [15-17]. Little is known about which types of tics are more suppressible, but one video analysis study of adolescents with TS showed that tic 
suppression was most effective in the body areas such as the trunk or proximal leg, and least effective in the hands and eyes, which had the most tics at baseline [18]. Although the sample size was small and types of tics were heterogeneous across the sample, this finding suggested that tic suppressibility follows a somatotopic pattern.

Tic suppression usually requires effort [12]. However, tic suppressibility can also occur to some extent without conscious awareness. In an observational study of children and adolescents with TS who were videotaped in the clinic and at home in three situations (holding a casual conversation with a stranger, being seated quietly with a stranger, and being seated quietly alone), the intensity and frequency of tics were highest when participants were alone at home and lowest when they were quietly seated with a stranger in the clinic (without conversing) [19]. Other observational studies of adults with TS showed tic frequency increased when they paid attention to their tics compared to when they were distracted from their tics (e.g., watching a video or performing a fine motor task), even when they did not intentionally suppress their tics $[20,21]$. These findings suggest that tic suppressibility may at times operate implicitly through modulation of attention to or distraction from the tics. This finding is important because it provides an explanation for the worsening or improvement of tics with changes in social context.

Tic suppressibility is also affected by the environment. A standardized tic suppression task (TST) was designed to characterize the effect of external environmental variables and quantify the ability to suppress tics under various conditions [16]. In this paradigm, participants are seated in front of a "tic detector" and are told either to tic freely 
or to suppress their tics. During the experiments, researchers in an adjacent room observe the participant's behavior through a one-way mirror or camera. They can then provide an immediate contingent reward for successful tic suppression in the form of a token dispensed from the tic detector remotely, which can be exchanged for money at the end of the study. Many studies using the TST paradigm in children with tics have demonstrated that adding rewards for tic-free periods to verbal instruction to suppress tics yielded greater reduction in tics than verbal instruction alone $[14,16,17,22,23]$. Further, contingency of the reward was important, as tokens delivered noncontingently did not show similar benefit on tic reduction [24]. A particularly clever study tested whether certain contexts could create an implicit stimulus for tic suppression [25]. Children with tics underwent the TST paradigm in the presence of different color lights (e.g., purple light presented when rewarded for tic suppression, orange light presented when verbally instructed to suppress tics without rewards). No explanation for the lights was provided, yet later, when participants were exposed to the light that had been associated with tic suppression during contingent reward, tic frequency was reduced even when no rewards or instruction to suppress tics were given. This study provides compelling evidence that implicitly learned environmental cues can affect tic suppressibility.

With years of experience suppressing tics, patients with tics may become better at tic suppression over time. Tic suppressibility is observable in children younger than 10 years old, but is correlated with age and frequency of tics [23, 26]. Still, somewhat surprisingly, children with recent onset of tics (i.e., within 6 months of tic onset) can suppress about $40 \%$ of their tics and do so more efficiently with immediate contingent 
reward [27]. Interestingly, those with better suppression in the presence of contingent reward showed better tic outcome (i.e., less tic severity) at a later timepoint, namely the one-year anniversary of tic onset [28]. Thus, tic suppressibility and its reinforceable nature may be inherent (or learned very early after the onset of tics), and this ability may be an important predictor of clinical outcomes.

Clinically, there was a concern that volitional tic suppression might elicit more tics once efforts to suppress stop, which was referred to as a "rebound effect." In fact, the Diagnostic Confidence Index, which summarizes classic features of TS, lists "Rebound effect after suppression" as one of the characteristic features [29]. A questionnaire survey of health care professionals including physicians and psychologists showed that $77 \%$ of them believed the rebound effect existed [30]. However, a number of clinical studies have shown no evidence of tics worsening after voluntary tic suppression, including with behavioral therapies for tics $[14,15,17,22,31]$. These results support the safety of behavioral therapies that employ tic suppression. However, tic suppression is not without potential negative consequences. More than $80 \%$ of TS patients reported that tic suppression caused fatigue, and $95 \%$ "felt uncomfortable or incomplete when they suppressed tics" [12]. Intentional tic suppression also re-allocates attentional resources, and many children with tics reported that tic suppression interfered with concentration at school [13]. A laboratory study supported this perception: children could suppress tics with or without a concomitant cognitive task, but performance on the task worsened during tic suppression [21]. Thus, effort expended suppressing tics may result in mental fatigue, which can affect social life, or may impair attention. Therefore, tic suppression needs to be 
advised judiciously, and ideally should be employed as part of an effective behavior therapy.

Another potential negative effect of tic suppression is that some children $(<10 \%)$ paradoxically have more tics when trying to suppress them [23]. This paradoxical increase in tics may stem from attempts to control premonitory urges. In the words of Franklin et al [32], "attempts to control aversive private events [like premonitory urges] tend to be ineffective and, paradoxically, increase the very thoughts and emotions that the individual was trying to avoid." Alternatively, "white knuckle" efforts to suppress tics may increase one's focus on the upcoming success or failure at tic suppression. Such a focus will often exacerbate anxiety, which in turn can exacerbate tics [33, 34]. In extreme cases, such internally-directed attention and worries about tics may lead to severe "tic attacks" [35]. Acceptance and Commitment Therapy [32] and cognitive-behavioral approaches to tics [36] address these internal factors. Since tics improve with distraction and worsen when focusing on them, distraction from tics may be an important part of such treatment.

\section{Mechanism/physiology of tic suppressibility}

The pathophysiology of TS has been described as involving development-related atypical functional brain connectivity and impaired function of cortical-striatal-thalamiccortical (CSTC) circuits with aberrant neurotransmitter function, including dopamine, 
serotonin, and GABA [9, 37-43]. Some structural and functional neuroimaging and neurophysiological studies have explored the neural correlates of tic suppressibility. An early functional magnetic resonance imaging (fMRI) study of adults with TS showed widespread signal intensity change (increases and decreases) during tic suppression in the basal ganglia, limbic system, thalamus, and a number of cortical regions, such as the frontal cortex, temporal gyrus, anterior cingulate gyrus, occipital cortex, and sensorimotor cortex [44]. The authors emphasized that subcortical-cortical circuits are involved in tic suppression. Although this study had limitations-e.g., decreased brain activity during tic suppression could simply reflect increased brain activity with tics - it illustrated that tic suppression involves broad cortical and subcortical networks.

Multiple neural circuits have been suggested to contribute to tic suppressibility. In the CSTC pathway, the frontal lobe plays an important role in executive functions [45]. In particular, a network including the SMA, inferior frontal cortex, basal ganglia and primary motor cortex has been suggested to be important for reactive stopping [46, 47]. Several tic suppression studies have focused on frontal regions. A task-based (suppression vs. free ticcing) fMRI experiment analyzed regional homogeneity (ReHO) in adults with TS [48]. $\mathrm{ReHO}$ is a measure of the functional connectivity of a region with its nearest neighbors. Tic suppression was associated with an increase in the ReHO of the inferior frontal gyrus, which was positively correlated with tic suppressibility [48]. An fMRI study comparing ocular tic suppression in TS patients and blink suppression in healthy controls showed the dorsal anterior cingulate cortex and associated limbic areas were activated during tic suppression, while the supplementary motor area (SMA), right ventrolateral prefrontal 
cortex and cingulate cortex were activated during blink suppression [49]. It is difficult to determine if these changes reflect brain activation of generating tics, performing tics, or suppressing tics. A novel event-related individualized model was designed recently that accounts for the transient decrease in PU after each accidental eye closure during blink tic suppression and allows for a tailor-made approach for future functional neuroimaging studies [50]. Applying this model to fMRI studies may help to identify BOLD activity specific to the urge to tic during tic suppression. Electroencephalography (EEG) studies of adults with TS suggest the involvement of premotor and prefrontal regions during tic suppression. Specifically, functional connectivity between the frontal and prefrontal cortices, and the sensorimotor cortex was noted during tic suppression [51, 52]. Another EEG study of adults with TS showed that abnormal beta activity in the sensorimotor cortex during the execution of tasks returned to its normal state during tic suppression [53]. These results could indicate that tic suppression exerts its effect by reorganizing abnormal overactivity of CSTC circuits that cause tics. In other words, tic suppression may involve mechanisms of top-down control operated by cortical regions connecting to and normalizing aberrant activity in subcortical structures.

The SMA may play a key role in the processes involved in suppressing tics. Previous human and animal fMRI studies suggested that the SMA is important for intended movement preparation and execution, but also for inhibitory motor control during motor imagery by suppressing premotor cortex activity when the movement is not to be performed [54-59]. Clinically, low frequency repetitive transcranial magnetic stimulation (rTMS) over the SMA significantly improved tic symptoms, measured by the Yale Global 
Tic Severity Scale (YGTSS) [60]. A number of neuroimaging studies in TS suggest a role of the SMA in tic suppression, even though they did not directly investigate tic suppression per se. For example, a magnetoencephalography (MEG) study with TS patients showed that the primary motor cortex was activated more during preparation and execution and then inhibited more after movement termination, which the authors argued might be influenced by tic suppression [61]. Subsequently, the same group provided evidence of augmented interactions between the SMA and primary motor cortex in TS patients [62]. An fMRI study using a stop signal reaction-time task showed that activation of the SMA was associated with successful stopping and correlated with tic frequency [63]. The authors proposed that the SMA is an important region for a global inhibitory mechanism for both voluntary motor control and tic suppression in TS. Another fMRI study using a Go/No-Go task showed less activation in the primary motor cortex and secondary motor areas including the SMA during the Go condition, which the authors interpreted as reflecting modulation of frontoparietal brain networks for motor and behavioral control in TS [64]. GABA concentration in the SMA was found to be inversely correlated with cortical excitability in the primary motor cortex and was associated with motor tic severity [65]. The authors suggested increased GABAergic tonic inhibition in the SMA results in enhanced control over volitional movements and suppression of tics [65]. Taken together, these studies revealed differences between individuals with and without TS that may relate to the control of tics. 


\section{Functional correlates of related symptoms}

\section{Premonitory urges and interoceptive awareness}

Many TS patients report that unpleasant somatic sensations increase before a tic and during tic suppression, and are momentarily alleviated by the performance of a tic $[9,66$, 67]. Studies using the TST paradigm showed that, acutely, the severity of PU was increased by tic suppression and decreased by discontinuation of tic suppression [68-70]. PU are closely associated with tics and have been considered as the driving force of tics [8]. The presence of PU also helps distinguish tics from other movement disorders. Studies using the YGTSS and the Premonitory Urge for Tics Scale (PUTS) demonstrated that the severity of PU was significantly correlated with tic severity in both adults and children with tic disorders $[71,72]$. Reductions in the severity of PU by behavioral therapies correlate with a decrease in tic severity [73]. However, some research on the relationship between PU and tic suppression has demonstrated different results. For example, behavioral therapies [74], cross sectional studies using questionnaires, rating scales such as YGTSS and PUTS, and video review [26, 67, 75, 76], and behavioral analyses using the TST paradigm [22, 77], showed no evidence of a correlation between tic suppressibility and PU severity. Thus, understanding the relationship between PU and tic suppressibility will be useful in improving current therapeutic approaches as well as developing new ones. In fact, 
identifying PU and separating them from tics is one component of behavioral therapies for tics [66].

Interoceptive awareness is awareness of internal physiological processes of receiving, accessing, and appraising internal bodily signals (e.g., visceral, vasomotor events) [78]. Based on the similarity between interoceptive awareness and PU, some studies have examined these phenomena, finding that higher severity of PU was associated with higher interoceptive awareness [79-80]. These results suggest that PU might represent enhanced or aberrant interoceptive sensations. However, no study has investigated the relationship between tic suppressibility and interoceptive awareness. Thus, future studies investigating such a relationship are clearly warranted.

\section{ADHD}

Attention-deficit hyperactivity disorder (ADHD) is common in TS patients. Similar to $\mathrm{TS}$, the pathophysiology of $\mathrm{ADHD}$ is thought to involve frontal-striatal networks, including the prefrontal cortex, cingulate cortex, and basal ganglia [81]. Previous studies support the idea that ADHD symptoms, especially attention problems, diminish tic suppressibility as well as the efficacy of behavioral therapy for tics. Laboratory-based studies using the TST paradigm, clinical data review, and a meta-analysis of inhibitory control in TS patients demonstrated that participants with comorbid tics and ADHD were capable of suppressing their tics, but those patients with more attention problems were less 
successful with this suppression and inhibitory control $[15,76,82]$. Moreover, TS patients with poorer response inhibition have been shown to respond less favorably to behavioral therapies for tics [83]. Improvement of tics has been seen during the treatment of ADHD. Methylphenidate (MPH) is a stimulant medicine for the treatment of ADHD and inhibits the reuptake of dopamine and norepinephrine [84]. Enhanced dopaminergic and noradrenergic activity in the prefrontal cortex may contribute to its efficacy in ADHD [85]. Clinical trials showed that tics improved significantly in those treated with MPH alone [86, 87]. Intriguingly, tic suppressibility did not improve with the one-time administration of DMPH although tics were decreased [87]. It is possible that improvement of tics with MPH is related to modulating the dopamine system as well as enhancing the ability to suppress tics with improvement in attention.

\section{Action inhibition}

As TS is related to difficulties preventing unwanted tic movements, some have hypothesized that the ability to control goal-directed movements is similarly affected. In fact, impaired action inhibition has been proposed as a key mechanism in TS [88]. However, various laboratory studies investigating tic suppressibility and inhibition of intentional action have yielded inconsistent findings [82]. For example, some studies using fMRI and EEG found no overall deficit in action inhibition in TS [51, 63]. On the other hand, some behavioral studies showed a paradoxical enhancement of cognitive inhibition over 
voluntary actions in TS [89-91]. This enhanced inhibition may be associated with compensatory adaptation to tics, including tic suppression.

Interestingly, a recent TMS study of adults with tic disorders found normal volitional movement preparation, execution, and inhibition, but impaired automatic (i.e., implicit or unconscious) action inhibition [92]. The authors suggested that tic disorders involve impairment of automatic action inhibition rather than volitional inhibition. However, a laboratory study using questionnaires (e.g., YGTSS) and a behavioral inhibition task showed no difference in automatic motor inhibition between patients with tics and healthy controls [93]. Additionally, adults with TS had reduced ability to suppress blinks, yet blink suppression and tic suppression were not correlated [50]. Given these mixed findings, further research is needed to elucidate the relationship between tic suppressibility and action inhibition.

\section{Conclusions}

This article discusses clinical features and neural correlates of tic suppressibility. As tic suppressibility is a distinct feature of tic disorders, a better understanding of it could provide new insight into the pathophysiology, prognosis, and treatment of tics. Current first-line behavioral interventions - exposure and response prevention (ERP) and habit reversal therapy (HRT) or its descendant Comprehensive Behavioral Interventions for Tics (CBIT) - are tic-suppression-based treatments. HRT employs awareness training and 
competing response training to encourage tic suppression for long durations of time [94]. ERP consists of exposure to stimuli that tend to induce tics, combined with practice to resist ticcing [95]. Both of these therapies lead eventually to diminished urges to tic and decreased tic frequency and severity.

Clinically, tic suppressibility seems to include both voluntary and involuntary components and is present at the beginning of the onset of tics, even at very young ages. Tic suppression can be modified by rewards or other environmental stimuli, and has been shown to predict future tic severity [28]. Thus, it is possible that early tic suppression can predict prognosis of tic disorders and/or response to treatment, a promising direction for future research.

While neurophysiological and neuroimaging research has demonstrated the involvement of different brain systems in tic suppression, there is little known about how these systems change over time. Longitudinal studies are necessary to improve our understanding of the natural course of tic suppressibility. Thus, additional measures of tic suppressibility may need to be developed for consistent assessment of the ability to suppress tics over development.

\section{Funding}

Research reported in this publication was supported by the National Institute of Mental Health of the National Institutes of Health under award numbers R01MH104030 and R01MH118217. The content is solely the responsibility of the authors and does not necessarily represent the official views of the National Institutes of Health. 


\section{Human and Animal Rights}

This article does not contain any studies with human or animal subjects performed by any of the authors. 


\section{References}

Papers of particular interest, published recently, have been highlighted as:

- Of importance

•• Of major importance

1. American Psychiatric Association. Diagnostic and statistical manual of mental disorders (DSM-5®). American Psychiatric Pub, 2013.

2. Snider LA, Seligman LD, Ketchen BR, Levitt SJ, Bates LR, Garvey MA, et al. prevalence, characterization, and associations. Pediatrics 2002;110:331-6. doi:10.1542/peds.110.2.331.

3. Kurlan R, McDermott MP, Deeley C, Como PG, Brower C, Eapen S, et al. Prevalence of tics in schoolchildren and association with placement in special education. Neurology 2001;57:1383-8. doi:10.1212/wnl.57.8.1383.

4. Black KJ, Black ER, Greene DJ, Schlaggar BL. Provisional Tic Disorder: What to tell parents when their child first starts ticcing. F1000Res 2016;5:696. doi:10.12688/f1000research.8428.1.

5. Leckman JF, Zhang H, Vitale A, Lahnin F, Lynch K, Bondi C, et al. Course of tic severity in Tourette syndrome: the first two decades. Pediatrics 1998;102:14-9. 
doi:10.1542/peds.102.1.14.

6. Mink JW. The Basal Ganglia and involuntary movements: impaired inhibition of competing motor patterns. Arch Neurol 2003;60:1365-8. doi:10.1001/archneur.60.10.1365.

7. Woods DW, Piacentini J, Himle MB, Chang S. Premonitory Urge for Tics Scale (PUTS): initial psychometric results and examination of the premonitory urge phenomenon in youths with Tic disorders. J Dev Behav Pediatr 2005;26:397-403.

doi:10.1097/00004703-200512000-00001.

8. Bliss J, Cohen DJ, Freedman DX. Sensory experiences of Gilles de la Tourette syndrome. Arch Gen Psychiatry 1980;37:1343-7.

doi:10.1001/archpsyc.1980.01780250029002.

9. Leckman JF. Tourette's syndrome. Lancet 2002;360:1577-86.

doi:10.1016/S0140-6736(02)11526-1.

10. Jankovic J. Tourette syndrome. Phenomenology and classification of tics. Neurol Clin 1997;15:267-75. doi:10.1016/s0733-8619(05)70311-x.

11. Koller WC, Biary NM. Volitional control of involuntary movements. Mov Disord 1989;4:153-6. doi:10.1002/mds.870040207.

12. Matsuda N, Kono T, Nonaka M, Fujio M, Kano Y. Self-initiated coping with Tourette's syndrome: Effect of tic suppression on QOL. Brain Dev 2016;38:233-41. doi:10.1016/j.braindev.2015.08.006.

13. Wadman R, Glazebrook C, Beer C, Jackson GM. Difficulties experienced by 
young people with Tourette syndrome in secondary school: a mixed methods description of self, parent and staff perspectives. BMC Psychiatry 2016;16:14. doi:10.1186/s12888-0160717-9.

14. Meidinger AL, Miltenberger RG, Himle M, Omvig M, Trainor C, Crosby R. An investigation of tic suppression and the rebound effect in Tourette's disorder. Behav Modif 2005;29:716-45. doi:10.1177/0145445505279262.

15. Himle MB, Woods DW. An experimental evaluation of tic suppression and the tic rebound effect. Behav Res Ther 2005;43:1443-51. doi:10.1016/j.brat.2004.11.002.

16. Woods DW, Himle MB. Creating tic suppression: comparing the effects of verbal instruction to differential reinforcement. J Appl Behav Anal 2004;37:417-20. doi:10.1901/jaba.2004.37-417.

17. Woods DW, Himle MB, Miltenberger RG, Carr JE, Osmon DC, Karsten AM, et al. Durability, negative impact, and neuropsychological predictors of tic suppression in children with chronic tic disorder. J Abnorm Child Psychol 2008;36:237-45. doi:10.1007/s10802-007-9173-9.

18. Ganos C, Bongert J, Asmuss L, Martino D, Haggard P, Münchau A. The somatotopy of tic inhibition: Where and how much?. Mov Disord 2015;30:1184-9. doi:10.1002/mds.26188.

19. Goetz CG, Leurgans S, Chmura TA. Home alone: methods to maximize tic expression for objective videotape assessments in Gilles de la Tourette syndrome. Mov 
Disord 2001;16:693-7. doi:10.1002/mds.1159.

20. Brandt VC, Lynn MT, Obst M, Brass M, Münchau A. Visual feedback of own tics increases tic frequency in patients with Tourette's syndrome. Cogn Neurosci 2015;6:1-7. doi:10.1080/17588928.2014.954990.

21. Misirlisoy E, Brandt V, Ganos C, Tübing J, Münchau A, Haggard P. The relation between attention and tic generation in Tourette syndrome. Neuropsychology 2015;29:658-65. doi:10.1037/neu0000161.

22. Specht MW, Woods DW, Nicotra CM, Kelly LM, Ricketts EJ, Conelea CA, et al. Effects of tic suppression: ability to suppress, rebound, negative reinforcement, and habituation to the premonitory urge. Behav Res Ther 2013;51:24-30.

doi:10.1016/j.brat.2012.09.009.

23. • Conelea CA, Wellen B, Woods DW, Greene DJ, Black KJ, Specht M, et al. Patterns and Predictors of Tic Suppressibility in Youth With Tic Disorders. Front Psychiatry 2018;9:188. doi:10.3389/fpsyt.2018.00188. This study includes the previous TST studies and discusses patterns and clinical correlates of reward-enhanced tic suppression.

24. Himle MB, Woods DW, Bunaciu L. Evaluating the role of contingency in differentially reinforced tic suppression. J Appl Behav Anal 2008;41:285-9. doi:10.1901/jaba.2008.41-285.

25. Woods DW, Walther MR, Bauer CC, Kemp JJ, Conelea CA. The development 
of stimulus control over tics: a potential explanation for contextually-based variability in the symptoms of Tourette syndrome. Behav Res Ther 2009;47:41-7. doi:10.1016/j.brat.2008.10.013.

26. Banaschewski T, Woerner W, Rothenberger A. Premonitory sensory phenomena and suppressibility of tics in Tourette syndrome: developmental aspects in children and adolescents. Dev Med Child Neurol 2003;45:700-3.

doi:10.1017/s0012162203001294.

27. Greene DJ, Koller JM, Robichaux-Viehoever A, Bihun EC, Schlaggar BL, Black KJ. Reward enhances tic suppression in children within months of tic disorder onset. Dev Cogn Neurosci 2015;11:65-74. doi:10.1016/j.den.2014.08.005.

28. - Kim S, Greene DJ, Robichaux-Viehoever A, et al. Tic Suppression in Children With Recent-Onset Tics Predicts 1-Year Tic Outcome. J Child Neurol 2019;34:757-64. doi:10.1177/0883073819855531. This study demonstrates prognostic value of tic suppression early after the onset of tics.

29. Robertson MM, Banerjee S, Kurlan R, Cohen DJ, Leckman JF, McMahon W, et al. The Tourette syndrome diagnostic confidence index: development and clinical associations. Neurology 1999;53:2108-12. doi:10.1212/wnl.53.9.2108.

30. Marcks BA, Woods DW, Teng EJ, Twohig MP. What do those who know, know? Investigating providers' knowledge about Tourette's Syndrome and its treatment. Cogn Behav Pract 2004;11:298-305. doi:10.1016/S1077-7229(04)80044-0. 
31. Verdellen CW, Hoogduin CA, Keijsers GP. Tic suppression in the treatment of Tourette's syndrome with exposure therapy: the rebound phenomenon reconsidered. Mov Disord 2007;22:1601-6. doi:10.1002/mds.21577.

32. Franklin ME, Best SH, Wilson MA, Loew B, Compton SN. Habit reversal training and acceptance and commitment therapy for Tourette syndrome: A pilot project. J Dev Phys Disabil 2011;23:49-60. doi:10.1007/s10882-010-9221-1.

33. O’Connor K, St-Pierre-Delorme MÈ, Leclerc J, Lavoie M, Blais MT. Metacognitions in Tourette syndrome, tic disorders, and body-focused repetitive disorder. Can J Psychiatry 2014;59:417-25. doi:10.1177/070674371405900804.

34. Himle MB, Capriotti MR, Hayes LP, Ramanujam K, Scahill L, Sukhodolsky DG, et al. Variables Associated With Tic Exacerbation in Children With Chronic Tic Disorders. Behav Modif 2014;38:163-83. doi:10.1177/0145445514531016.

35. Robinson S, Hedderly T. Novel Psychological Formulation and Treatment of "Tic Attacks" in Tourette Syndrome. Front Pediatr 2016;4:46. doi:10.3389/fped.2016.00046.

36. Leclerc JB, O’Connor KP, J.-Nolin G, Valois P, Lavoie ME. The Effect of a New Therapy for Children with Tics Targeting Underlying Cognitive, Behavioral, and Physiological Processes. Front Psychiatry 2016;7:135. doi:10.3389/fpsyt.2016.00135.

37. Peterson BS, Thomas P, Kane MJ, Scahill L, Zhang H, Bronen R, et al. Basal Ganglia volumes in patients with Gilles de la Tourette syndrome. Arch Gen Psychiatry 
2003;60:415-24. doi:10.1001/archpsyc.60.4.415.

38. •- Augustine F, Singer HS. Merging the Pathophysiology and Pharmacotherapy of Tics. Tremor Other Hyperkinet Mov (N Y). 2019;8:595. doi:10.7916/D8H14JTX. A useful review of the pathophysiology and current pharmacological treatment of tics.

39. Church JA, Fair DA, Dosenbach NU, Cohen AL, Miezin FM, Petersen SE, et al. Control networks in paediatric Tourette syndrome show immature and anomalous patterns of functional connectivity. Brain 2009;132:225-38. doi:10.1093/brain/awn223.

40. Yael D, Vinner E, Bar-Gad I. Pathophysiology of tic disorders. Mov Disord 2015;30:1171-8. doi:10.1002/mds.26304.

41. Albin RL, Mink JW. Recent advances in Tourette syndrome research. Trends Neurosci 2006;29:175-82. doi:10.1016/j.tins.2006.01.001.

42. Singer HS. Tourette's syndrome: from behaviour to biology. Lancet Neurol 2005;4:149-59. doi:10.1016/S1474-4422(05)01012-4.

43. - Nielsen AN, Gratton C, Church JA, Dosenbach NUF, Black KJ, Petersen SE, et al. Atypical Functional Connectivity in Tourette Syndrome Differs Between Children and Adults. Biol Psychiatry 2020;87:164-73. doi:10.1016/j.biopsych.2019.06.021. This study demonstrates altered brain networks in patietns with tics.

44. Peterson BS, Skudlarski P, Andersen AW, Zhang H, Gatenby JC, Lacadie CM, et al. A functional magnetic resonance imaging study of tic suppression in Tourette 
syndrome. Arch Gen Psychiatry 1998;55:326-33. doi:10.1001/archpsyc.55.4.326.

45. Alvarez JA, Emory E. Executive function and the frontal lobes: a meta-analytic review. Neuropsychol Rev 2006;16:17-42. doi:10.1007/s11065-006-9002-x.

46. Aron AR. From reactive to proactive and selective control: developing a richer model for stopping inappropriate responses. Biol Psychiatry 2011;69:e55-e68. doi:10.1016/j.biopsych.2010.07.024.

47. Swick D, Ashley V, Turken AU. Left inferior frontal gyrus is critical for response inhibition. BMC Neurosci 2008;9:102. doi:10.1186/1471-2202-9-102.

48. Ganos C, Kahl U, Brandt V, Schunke O, Bäumer T, Thomalla G, et al. The neural correlates of tic inhibition in Gilles de la Tourette syndrome. Neuropsychologia 2014;65:297-301. doi:10.1016/j.neuropsychologia.2014.08.007.

49. van der Salm SMA, van der Meer JN, Cath DC, Groot PFC, van der Werf YD, Brouwers E, et al. Distinctive tics suppression network in Gilles de la Tourette syndrome distinguished from suppression of natural urges using multimodal imaging. Neuroimage Clin 2018;20:783-92. doi:10.1016/j.nicl.2018.09.014.

50. Botteron HE, Richards CA, Nishino T, Ueda K, Acevedo HK, Koller JM, et al.

The urge to blink in Tourette syndrome. Cortex 2019;120:556-66. doi:10.1016/j.cortex.2019.07.010. This study proposes a novel event-related individualized model to explore BOLD activity specific to the urge to tic during tic suppression. 
51. Serrien DJ, Orth M, Evans AH, Lees AJ, Brown P. Motor inhibition in patients with Gilles de la Tourette syndrome: functional activation patterns as revealed by EEG coherence. Brain 2005;128:116-25. doi:10.1093/brain/awh318.

52. Hong HJ, Sohn H, Cha M, Kim S, Oh J, Chu MK, et al. Increased frontomotor oscillations during tic suppression in children with Tourette syndrome. J Child Neurol 2013;28:615-24. doi:10.1177/0883073812450317.

53. - Zapparoli L, Macerollo A, Joyce EM, Martino D, Kilner JM. Voluntary tic suppression and the normalization of motor cortical beta power in Gilles de la Tourette syndrome: an EEG study. Eur J Neurosci 2019;50:3944-57. doi:10.1111/ejn.14548. This paper explores neuronal activity over the sensorimotor cortex during involuntary movements and tic suppression.

54. Wardak C. The Role of the Supplementary Motor Area in Inhibitory Control in Monkeys and Humans. J Neurosci 2011;31:5181-3. doi:10.1523/JNEUROSCI.000611.2011.

55. Dinomais M, Minassian AT, Tuilier T, Delion M, Wilke M, N'Guyen S, et al. Functional MRI comparison of passive and active movement: possible inhibitory role of supplementary motor area. Neuroreport 2009;20:1351-5.

doi:10.1097/WNR.0b013e328330cd43.

56. Kasess CH, Windischberger C, Cunnington R, Lanzenberger R, Pezawas L, Moser E. The suppressive influence of SMA on M1 in motor imagery revealed by fMRI 
and dynamic causal modeling. Neuroimage 2008;40:828-37.

doi:10.1016/j.neuroimage.2007.11.040.

57. Scangos KW, Stuphorn V. Medial frontal cortex motivates but does not control movement initiation in the countermanding task. J Neurosci 2010;30:1968-82. doi:10.1523/JNEUROSCI.4509-09.2010.

58. Chen X, Scangos KW, Stuphorn V. Supplementary motor area exerts proactive and reactive control of arm movements. J Neurosci 2010;30:14657-75. doi:10.1523/JNEUROSCI.2669-10.2010.

59. Duann JR, Ide JS, Luo X, Li CS. Functional connectivity delineates distinct roles of the inferior frontal cortex and presupplementary motor area in stop signal inhibition. J Neurosci 2009;29:10171-9. doi:10.1523/JNEUROSCI.1300-09.2009.

60. Mantovani A, Lisanby SH, Pieraccini F, Ulivelli M, Castrogiovanni P, Rossi S. Repetitive transcranial magnetic stimulation (rTMS) in the treatment of obsessivecompulsive disorder (OCD) and Tourette's syndrome (TS). Int J Neuropsychopharmacol 2006;9:95-100. doi:10.1017/S1461145705005729.

61. Franzkowiak S, Pollok B, Biermann-Ruben K, Südmeyer M, Paszek J, Jonas M, et al. Altered pattern of motor cortical activation-inhibition during voluntary movements in Tourette syndrome. Mov Disord 2010;25:1960-6. doi:10.1002/mds.23186.

62. Franzkowiak S, Pollok B, Biermann-Ruben K, Südmeyer M, Paszek J, Thomalla G, et al. Motor-cortical interaction in Gilles de la Tourette syndrome. PLoS One 
2012;7:e27850. doi:10.1371/journal.pone.0027850.

63. Ganos C, Kühn S, Kahl U, Schunke O, Feldheim J, Gerloff C, et al. Action inhibition in Tourette syndrome. Mov Disord 2014;29:1532-8. doi:10.1002/mds.25944.

64. Thomalla G, Jonas M, Bäumer T, Siebner HR, Biermann-Ruben K, Ganos C, et al. Costs of control: decreased motor cortex engagement during a Go/NoGo task in Tourette's syndrome. Brain 2014;137:122-36. doi:10.1093/brain/awt288.

65. Draper A, Stephenson MC, Jackson GM, Pépés S, Morgan PS, Morris PG, et al. Increased GABA contributes to enhanced control over motor excitability in Tourette syndrome. Curr Biol 2014;24:2343-7. doi:10.1016/j.cub.2014.08.038.

66. Brandt VC, Beck C, Sajin V, Baaske MK, Bäumer T, Beste C, et al. Temporal relationship between premonitory urges and tics in Gilles de la Tourette syndrome. Cortex 2016;77:24-37. doi:10.1016/j.cortex.2016.01.008.

67. Müller-Vahl KR, Riemann L, Bokemeyer S. Tourette patients' misbelief of a tic rebound is due to overall difficulties in reliable tic rating. J Psychosom Res 2014;76:472-6. doi:10.1016/j.jpsychores.2014.03.003.

68. Himle MB, Woods DW, Conelea CA, Bauer CC, Rice KA. Investigating the effects of tic suppression on premonitory urge ratings in children and adolescents with Tourette's syndrome. Behav Res Ther 2007;45:2964-76. doi:10.1016/j.brat.2007.08.007.

69. Capriotti MR, Brandt BC, Turkel JE, Lee HJ, Woods DW. Negative Reinforcement and Premonitory Urges in Youth With Tourette Syndrome: An 
Experimental Evaluation. Behav Modif 2014;38:276-96. doi:10.1177/0145445514531015.

70. Specht MW, Nicotra CM, Kelly LM, Woods DW, Ricketts EJ, Perry-Parrish C, et al. A Comparison of Urge Intensity and the Probability of Tic Completion During Tic Freely and Tic Suppression Conditions. Behav Modif 2014;38:297-318. doi: $10.1177 / 0145445514537059$.

71. Draper A, Jackson GM, Morgan PS, Jackson SR. Premonitory urges are associated with decreased grey matter thickness within the insula and sensorimotor cortex in young people with Tourette syndrome. J Neuropsychol 2016;10:143-53. doi:10.1111/jnp.12089.

72. • Kyriazi M, Kalyva E, Vargiami E, Krikonis K, Zafeiriou D. Premonitory Urges and Their Link With Tic Severity in Children and Adolescents With Tic Disorders. Front Psychiatry 2019;10:569. doi:10.3389/fpsyt.2019.00569. This study explored relationship between premonitory urge and tic severity.

73. Verdellen CW, Hoogduin CA, Kato BS, Keijsers GP, Cath DC, Hoijtink HB. Habituation of premonitory sensations during exposure and response prevention treatment in Tourette's syndrome. Behav Modif 2008;32:215-27. doi:10.1177/0145445507309020.

74. Houghton DC, Capriotti MR, Scahill LD, Wilhelm S, Peterson AL, Walkup JT, et al. Investigating Habituation to Premonitory Urges in Behavior Therapy for Tic Disorders. Behav Ther 2017;48:834-46. doi:10.1016/j.beth.2017.08.004.

75. Ganos C, Kahl U, Schunke O, Kühn S, Haggard P, Gerloff C, et al. Are 
premonitory urges a prerequisite of tic inhibition in Gilles de la Tourette syndrome?. J Neurol Neurosurg Psychiatry. 2012;83:975-8. doi:10.1136/jnnp-2012-303033.

76. Sambrani T, Jakubovski E, Müller-Vahl KR. New Insights into Clinical Characteristics of Gilles de la Tourette Syndrome: Findings in 1032 Patients from a Single German Center. Front Neurosci 2016;10:415. doi:10.3389/fnins.2016.00415.

77. Conelea CA, Woods DW, Brandt BC. The impact of a stress induction task on tic frequencies in youth with Tourette Syndrome. Behav Res Ther 2011;49:492-7. doi:10.1016/j.brat.2011.05.006.

78. Craig AD. How do you feel--now? The anterior insula and human awareness. Nat Rev Neurosci 2009;10:59-70. doi:10.1038/nrn2555.

79. Jackson SR, Parkinson A, Kim SY, Schüermann M, Eickhoff SB. On the functional anatomy of the urge-for-action. Cogn Neurosci 2011;2:227-43. doi:10.1080/17588928.2011.604717.

80. Ganos C, Garrido A, Navalpotro-Gómez I, Ricciardi L, Martino D, Edwards MJ, et al. Premonitory urge to tic in Tourette's is associated with interoceptive awareness. Mov Disord 2015;30:1198-202. doi:10.1002/mds.26228.

81. Curatolo P, D'Agati E, Moavero R. The neurobiological basis of ADHD. Italian Journal of Pediatrics 2010;36:79. doi:10.1186/1824-7288-36-79.

82. Morand-Beaulieu S, Grot S, Lavoie J, Leclerc JB, Luck D, Lavoie ME. The puzzling question of inhibitory control in Tourette syndrome: A meta-analysis. Neurosci 
Biobehav Rev 2017;80:240-62. doi:10.1016/j.neubiorev.2017.05.006.

83. Deckersbach T, Rauch S, Buhlmann U, Wilhelm S. Habit reversal versus supportive psychotherapy in Tourette's disorder: a randomized controlled trial and predictors of treatment response. Behav Res Ther 2006;44:1079-90.

doi:10.1016/j.brat.2005.08.007.

84. Challman TD, Lipsky JJ. Methylphenidate: its pharmacology and uses. Mayo Clin Proc 2000;75:711-21. doi:10.4065/75.7.711.

85. Arnsten AF, Dudley AG. Methylphenidate improves prefrontal cortical cognitive function through alpha2 adrenoceptor and dopamine D1 receptor actions: Relevance to therapeutic effects in Attention Deficit Hyperactivity Disorder. Behav Brain Funct 2005;1:2. doi:10.1186/1744-9081-1-2.

86. Tourette's Syndrome Study Group. Treatment of ADHD in children with tics: a randomized controlled trial. Neurology 2002;58:527-36. doi:10.1212/wnl.58.4.527.

87. Lyon GJ, Samar SM, Conelea C, Trujillo MR, Lipinski CM, Bauer CC, et al. Testing tic suppression: comparing the effects of dexmethylphenidate to no medication in children and adolescents with attention-deficit/hyperactivity disorder and Tourette's disorder. J Child Adolesc Psychopharmacol 2010;20:283-9. doi:10.1089/cap.2010.0032.

88. Stern ER, Blair C, Peterson BS. Inhibitory deficits in Tourette's syndrome. Dev Psychobiol 2008;50:9-18. doi:10.1002/dev.20266.

89. Jackson GM, Mueller SC, Hambleton K, Hollis CP. Enhanced cognitive control 
in Tourette Syndrome during task uncertainty. Exp Brain Res 2007;182:357-64. doi:10.1007/s00221-007-0999-8.

90. Jackson SR, Parkinson A, Jung J, Ryan SE, Morgan PS, Hollis C, et al. Compensatory neural reorganization in Tourette syndrome. Curr Biol 2011;21:580-5. doi:10.1016/j.cub.2011.02.047.

91. Mueller SC, Jackson GM, Dhalla R, Datsopoulos S, Hollis CP. Enhanced cognitive control in young people with Tourette's syndrome. Curr Biol 2006;16:570-3. doi:10.1016/j.cub.2006.01.064.

92. • Rawji V, Modi S, Latorre A, Rocchi L, Hockey L, Bhatia K, et al. Impaired automatic but intact volitional inhibition in primary tic disorders. Brain 2020;143:906-919. doi:10.1093/brain/awaa024. This paper looks at automatic inhibition in TS patients.

93. Stenner MP, Baumgaertel C, Heinze HJ, Ganos C, Müller-Vahl KR. Intact automatic motor inhibition in patients with Tourette syndrome. Mov Disord 2018;33:18004. doi:10.1002/mds.27493.

94. Piacentini J, Woods DW, Scahill L, Wilhelm S, Peterson AL, Chang S, et al. Behavior therapy for children with Tourette disorder: a randomized controlled trial. JAMA 2010;303:1929-37. doi:10.1001/jama.2010.607.

95. Verdellen CW, Keijsers GP, Cath DC, Hoogduin CA. Exposure with response prevention versus habit reversal in Tourettes's syndrome: a controlled study. Behav Res Ther 2004;42:501-11. doi:10.1016/S0005-7967(03)00154-2. 